\title{
Multimodal genetic features correlate with poor response to neoadjuvant chemoradiotherapy and high recurrence risk in Chinese patients with stage IB-IIA cervical cancer
}

\author{
Yuchun Wei \\ Shandong Cancer Hospital affiliated to Shandong University \\ Chuqing Wei \\ Shandong Cancer Hospital: Shandong Tumor Hospital and Institute \\ Chen Liang \\ Shandong Cancer Hospital: Shandong Tumor Hospital and Institute \\ Ning Liu \\ Shandong Cancer Hospital: Shandong Tumor Hospital and Institute \\ Zhenhao Fang \\ Nanjing Geneseeq Technology Inc.
}

Jiani C. Yin

Nanjing Geneseeq Technology Inc.

Jiaohui Pang

Nanjing Geneseeq Technology Inc.

Qiuxiang Ou

Nanjing Geneseeq Technology Inc.

Xue Wu

Nanjing Geneseeq Technology Inc.

Xiaonan Wang

Nanjing Geneseeq Technology Inc.

Dianbin Mu

Shandong Cancer Hospital: Shandong Tumor Hospital and Institute

Yang Shao

Nanjing Institute of Technology Inc.

Jinming Yu

Shandong Cancer Hospital: Shandong Tumor Hospital and Institute

Shuanghu Yuan ( $\sim$ yuanshuanghu@sina.com )

Shandong Cancer Hospital: Shandong Tumor Hospital and Institute https://orcid.org/0000-0002-83272524 
Research

Keywords: Cervical Cancer, Neoadjuvant chemoradiotherapy, Pathological response, Disease free survival, Ethnicity specific mutations

Posted Date: October 2nd, 2020

DOI: https://doi.org/10.21203/rs.3.rs-84299/v1

License: (c) (i) This work is licensed under a Creative Commons Attribution 4.0 International License.

Read Full License 


\section{Abstract}

Background Response of cervical cancer patients to neoadjuvant therapy differs from person to person. It remains unclear whether genetic alterations can predict response to neoadjuvant therapy and diseasefree survival in cervical cancer.

Methods 62 Chinese patients with stage IB-IIA cervical cancer were included in this study. Pre-treatment tumor tissues were profiled using a targeted next-generation sequencing assay. Genetic alterations were compared with those identified in the Western populations using the TCGA database. Pathological response and disease-free survival (DFS) were evaluated and their correlations with genetic alterations were analyzed.

Results Genetic alterations in PIK3CA were prevalent in both Chinese and Caucasian populations. The mutation frequencies of TERT, POLD1, NOS2, and FGFR3 were significantly higher in Chinese patients whereas RPTOR, EGFR, and TP53 were frequently mutated in Caucasian patients. Germline mutations were identified in 13 out of 62 Chinese patients and $57 \%$ of them occurred in DNA repair genes, such as BRCA1/2, TP53 and PALB2. High tumor mutation burden (TMB), TP53 polymorphism (rs1042522) and $K E A P 1$ mutations were found to be associated with poor response to neoadjuvant therapy. KEAP1 mutations, PIK3CA-SOX2 co-amplification, TERC amplification and TYMS polymorphism were associated with higher relapse rates of cervical cancer.

Conclusion The similarity and difference of mutation landscape of Chinese and Caucasian patients suggested genetic background played a role in shaping the architecture of cervical cancer mutations. The associations of mutation feature of cervical cancer with patient response and tumor recurrence risk provided rationale to further validate and explore potential biomarkers for cervical cancer patients.

\section{Introduction}

Cervical cancer is the fourth most common cancer diagnosed among females and every year leads to more than half-million new cases as well as over 300,000 deaths worldwide [1]. Despite recent advances in prevention, diagnosis and treatment, clinical outcome of cervical cancer patients remains poor in the developing countries[2]. While the incidence of cervical cancer in developed countries has more than halved over the past decades, a surge in cervical cancer incidence was recently reported in China[3]. On the macro level, insufficient pap smear screening and HPV vaccination are the major culprits of this international disparity. On the molecular level, there may also exist differences in the mutational landscape of cervical cancer between Chinese and the Western populations, which may reveal clues of carcinogenesis mechanism and susceptibility between the two populations.

The primary treatment strategy for patients with early-stage cervical cancers, particularly stage IA-IB1, is radical hysterectomy with or without radiation or chemotherapy[2, 4]. Multiple treatment regimens have been actively explored and proposed for high-risk early-stage (stage IB-IIA) cervical cancer patients[5, 6]. Neoadjuvant brachytherapy and chemotherapy followed by radical surgery showed an efficacy non- 
inferior to standard chemoradiation treatment and a more favorable toxicity profile in stage IB2-IIA cervical cancer[6]. Despite high disease-free survival rate (90\% during 52 months of median follow-up time), there is a portion of patients who failed to respond to the therapy. Identification of potential biomarkers predicting poor treatment response in these patients is much needed.

In this study, we compared the genetic landscape of cervical cancer between Chinese and the Western populations to understand the differences in potential tumorigenesis mechanisms and identified associations between specific genetic alterations and poor treatment response to neoadjuvant therapy.

\section{Methods}

\section{Patients and study design}

Patients were enrolled according to the following criteria: 1) cervical cancer patients with histologically confirmed International Federation of Gynecology and Obstetrics (FIGO) stage IB1-IIA; 2) age $\geq 18$ years old; 3 ) pathological subtypes were squamous cell carcinoma, adenocarcinoma or adenosquamous carcinoma, excluding special types of tumors, such as clear cells carcinoma; 4) Eastern Cooperative Oncology Group performance status score of $0-2 ; 5)$ Patients voluntarily joined this study, signed informed consent and provided diagnosis and treatment data after cancer diagnosis before entering the group, good compliance, and cooperation with follow-up. Among the 62 patients, five of them dropped out during follow up after surgery due to loss of contact or withdrawal of consent.

Exclusion criteria: 1) potential radiation field overlap caused by previous radiotherapy; 2) patients cannot undergo routine imaging examination; 3 ) any signs of severe or uncontrolled systemic diseases that the researchers believe may significantly affect the patient's risk / benefit balance, including hepatitis $B$, hepatitis $\mathrm{C}$ and human immunodeficiency virus;

\section{Treatment}

The patients received one cycle of paclitaxel + cisplatin chemotherapy and brachytherapy ((500-700) cGy $\times(1-2)$ fraction) before operation, radical cervical cancer resection (extensive hysterectomy + pelvic lymph node dissection \pm adnexal lymphadenectomy \pm abdominal para-aortic lymphadenectomy). The radical surgery was followed by adjuvant chemo (three cycles), brachytherapy and irradiation (5040 cGy/28 fraction).

\section{Samples And Clinical Data}

The detailed clinical data of the selected patients were recorded, including age, pathological grade, imaging examination (CT, MRI or PET-CT, etc.) with or without lymph node metastasis, tumor stage, immunohistochemical results, course of disease, location and size of lesions, performance status score, family history. Paraffin samples of tumors were biopsied before and after radiotherapy and chemotherapy for next-generation sequencing and pathological response assessment, respectively. $10 \mathrm{ml}$ of venous blood was collected from each patient after chemo-radiotherapy and was kept in the purple lid 
EDTA anticoagulant blood collection tube (BD Vacutainer, Cat \# 367525). The white blood cell or normal tissue adjacent to tumor was used as control of tumor samples.

\section{Pathological Assessments}

The tumor samples were taken and subjected to Hematoxylin and eosin staining protocol after chemoradiotherapy to evaluate their pathological response to treatment. Hematoxylin and eosin-stained slides of sections of tumors after treatment were evaluated by pathologists blinded to the patient information. At least 1 section was taken every centimeter of tumor along its greatest diameter. 5 to 30 slides were examined for each case. The residual tumor percentage was quantified by dividing the estimated cross-sectional area of the viable tumor by total cross-sectional areas evaluated on each slide. Histologic parameters analyzed include inflammation, necrosis, fibrosis, giant cell reaction, foamy macrophages, and cholesterol cleft granuloma. The mean values of viable tumor cells for each patient were calculated by averaging the results for all slides.

\section{Sample Collection And Library Preparation}

Sample processing and genomic profiling were performed in a CLIA- and CAP-accredited laboratory (Nanjing Geneseeq Technology Inc., Nanjing, China) as previously described[7]. In brief, genomic DNA from tumor specimen and control samples were extracted and quantified by Qubit 3.0. Library preparations were performed with KAPA Hyper Prep Kit (KAPA Biosystems, USA). Target enrichment was performed using customized xGen lockdown probes (Integrated DNA Technologies) targeting 474 cancerand radiotherapy response-relevant genes (Radiotron ${ }^{\circledR}$, Nanjing Geneseeq Technology Inc., Nanjing). The hybridization capture reaction was performed with Dynabeads M-279 (Life Technologies) and xGen Lockdown hybridization and wash kit (Integrated DNA Technologies) according to manufacturer's protocols. Captured libraries were on-beads PCR amplified with Illumina p5 and p7 primers in KAPA HiFi HotStart ReadyMix (KAPA Biosystems), followed by purification using Agencourt AMPure XP beads. Libraries were quantified by qPCR using KAPA Library Quantification kit (KAPA Biosystems). Library fragment size was determined by Bioanalyzer 2100 (Agilent Technologies).

\section{Targeted Next-Generation Sequencing And Data Processing}

Sequencing was performed on the Illumina HiSeq4000 platform followed by data analysis as previously described[8, 9]. In brief, sequencing data were analyzed by Trimmomatic[10] to remove low-quality (quality < 15) or $\mathrm{N}$ bases, and then mapped to the human reference genome hg19 using the BurrowsWheeler Aligner (https://github.com/lh3/bwa/tree/master/bwakit). PCR duplicates were removed by Picard (available at: https://broadinstitute.github.io/picard/). The Genome Analysis Toolkit (GATK) (https://software.broadinstitute.org/gatk/) was used to perform local realignments around indels and base quality reassurance. SNPs and indels were analyzed by VarScan2[11] and HaplotypeCaller/UnifiedGenotyper in GATK, with the mutant allele frequency (MAF) cutoff as $0.5 \%$ for tissue samples, $0.1 \%$ for cfDNA samples, and a minimum of three unique mutant reads. Common SNPs were excluded if they were present in > 1\% population frequency in the 1000 Genomes Project or the 
Exome Aggregation Consortium (ExAC) 65000 exomes database. The resulting mutation list was further filtered by an in-house list of recurrent artifacts based on a normal pool of whole blood samples. Gene fusions were identified by FACTERA[12].

Tumor mutation burden (TMB) was calculated based on the number of non-silent somatic mutations per megabase coding region sequenced[7]. Microsatellite (MS) status of tumor sample was determined on the overall stability of MS loci tested in the panel. A sample was reported as microsatellite instable ("MSI") if $\geq 40 \%$ of the MS loci display instability, or as "MSS" if $<40 \%$ of the MS loci display instability.

\section{Statistical analysis}

Disease-free survival (DFS) was defined as the time from neoadjuvant chemoradiotherapy until time of the tumor relapse or date of last follow-up (May 5, 2020). A p-value of less than 0.05 was taken to be significant. The statistical analyses were performed using R (version 3.4.2). Gene pathways were analyzed using ReactomePA R package[13].

\section{Results}

\section{Patient clinical characteristics}

A total of 62 patients with stage $\varangle \mathrm{B}-\varangle \mathrm{A}$ cervical cancer diagnosed in Shandong Cancer Hospital from 2016 to 2019 were enrolled in this study and received neoadjuvant chemoradiotherapy plus radical hysterectomy (Table 1). The median age of patients was 47 years, ranging from 26 to 66 years. $55 \%$ of patients presented with stage IB disease. Squamous cell carcinoma was the most common histological subtype (82\%). The rest were adenocarcinoma (15\%) and adenosquamous carcinoma. As of May 5 , 2020 , the median follow-up time was 31 months. Three patients' cervical cancer relapsed during followup and the remaining $95 \%$ patients were relapse-free. 
Table 1. Clincal characteristics of cervical cancer patients

\section{Chinese $(n=62)$ Caucasian $(n=82)$}

Age (years)

$>44$

$35(56 \%)$

$46(56 \%)$

Median

47

45

Range

$26-66$

20-80

Clinical stage

IB

$34(55 \%)$

$72(88 \%)$

$\square \mathrm{A}$

$28(45 \%)$

$10(12 \%)$

Histological type

Squamous cell carcinoma $51(82 \%) \quad 61(74 \%)$

Others

$11(18 \%)$

$21(26 \%)$

Viable tumor cells

$<10 \%$

$13(21 \%)$

NA

$10 \%-50 \%$

$22(35 \%)$

NA

$50 \%-100 \%$

$27(44 \%)$

NA

Note: Caucasian patient data were derived from TCGA database

Table 2. Germline mutant patient characteristics

\begin{tabular}{lllllll}
\hline Patient ID & Age & Stage & Histology & Gene & AAchange2 & variant_type \\
\hline CC_006 & 35 & IIA & ADC & MPL & W398X & Nonsense variant \\
CC_007 & 44 & IIA & SCC & MMP1 & A330LfSX45 & Frame shift variant \\
\hline CC_015 & 41 & IB & SCC & PMS1 & K894RfsX17 & Frame shift variant \\
\hline CC_019 & 36 & IB & SCC & AXIN2 & R714W & Missense variant \\
\hline CC_028 & 60 & IB & SCC & BRCA1 & c.4358-2A>G & Splice variant \\
\hline CC_028 & 60 & IB & SCC & BRIP1 & S618* & Nonsense variant \\
\hline CC_034 & 66 & IB & SCC & EPCAM & L78R & Missense variant \\
\hline CC_036 & 59 & IIA & SCC & PALB2 & S537L & Missense variant \\
\hline CC_039 & 50 & IB & SCC & MUTYH & Y453C & Missense variant \\
\hline CC_039 & 50 & IB & SCC & TP53 & A86V & Missense variant \\
\hline CC_056 & 43 & IIA & SCC & BRCA2 & S2414* & Nonsense variant \\
\hline CC_072 & 38 & IB & ADC & FANCE & S157Kfs*21 & Frame shift variant \\
\hline CC_079 & 41 & IIA & SCC & BRCA1 & S451Lfs*20 & Frame shift variant \\
\hline CC_081 & 44 & IB & ADC & MLH1 & S295G & Missense variant \\
\hline CC_111 & 42 & IB & SCC & FANCM & L923Cfs*3 & Frame shift variant \\
\hline
\end{tabular}

To explore potential factors predicting cancer recurrence, pathological response of cervical cancer to neoadjuvant chemoradiotherapy was determined. $56 \%$ and $21 \%$ patient tumor samples showed partial pathological response ( $<50 \%$ viable tumor cells) and major pathological response $(<10 \%$ viable tumor 
cells)[14] to neoadjuvant therapy, respectively. All three patients with progressive disease after surgery failed to have major pathological response, indicating resistance to chemoradiotherapy was one of the factors promoting cancer relapse.

\section{Molecular Characteristics Of Cervical Cancer}

To find underlying signal pathways associated with cancer recurrence and resistance to chemoradiotherapy, we performed mutation profiling using targeted next-generation sequencing. First, we characterized the mutational landscape of cervical cancer to identify potential genetic alterations underlying disease pathogenesis. The top 29 genes most frequently mutated among the 62 Chinese patients with cervical cancer were shown in Fig. 1. The three genes with the highest mutation frequencies were PIK3CA, TERT and PKHD1. To compare the ethnical difference in mutation frequency of cervical cancer, a total of 82 Caucasian patients with stage $\varangle \mathrm{B}-\varangle \mathrm{A}$ cervical cancer in the TCGA database were analyzed [15] (Table 1).

Compared to other genes, the PIK3CA mutation rate was highest in cervical cancer of Chinese and Caucasian. PIK3CA mutations accounted for over one-third of all mutations found in cervical cancer in both populations (Table S1). More than half of them occurred on three hot spots in exon9 and 20, including E542, E545, and $\mathrm{H} 1047$, which were involved in inhibitory interaction with regulatory subunit (E542 and E545) and membrane association (H1047)[16]. About a quarter of PIK3CA alterations were amplification. Three patients carried two types of gene variations in PIK3CA, including E365K-E545K double mutation, amplification with S306C single mutation or E542K-E545K double mutation. Alteration frequency of genes in cervical cancer was compared among Chinese and Caucasian patients, as well as among different histological subtypes. TERT, POLD1, NOS2 and FGFR3 genes were frequently altered in Chinese cervical cancer, whereas RPTOR, EGFR and TP53 gene variants were significantly enriched in Caucasian cervical cancer (Figure S1A). ARID1A, PTEN and CTNNB1 gene mutations were frequently observed in cervical adenocarcinoma (Figure S1B).

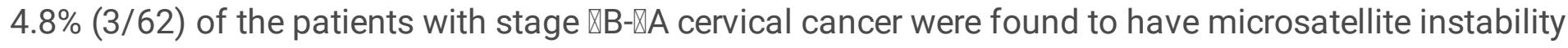
(MSI). Tumor mutation burden (TMB) in patients with MSI or microsatellite stability (MSS) was shown in Figure S2A. The upper tertile in the TMB distribution of all patients was defined as TMB-high (11.5 mutations/Mb) in this study. All the patients in MSI group had high TMB. No significant difference in TMB was observed between squamous cell carcinoma and adenocarcinoma (Figure S2B).

Germline mutations were detected in $21 \%$ of patients (13/62) (Table 2). Among the 14 genes carrying germline mutations, 8 genes including TP53, BRCA2, BRIP1, BRCA1, FANCM, MUTYH, FANCE and PALB2, were associated with DNA-repair pathway and half of them (BRCA2, BRIP1, BRCA1 and PALB) were involved in homology-directed repair (Table 2). In this study, the average diagnosed age of patients with germline mutations was 43 years old, 6 years younger than that of patients without germline mutations. $62 \%(7 / 13)$ germline-mutant cancer was stage IB. The majority $(77 \%)$ of patients had squamous cell carcinoma. Among the germline mutations harbored by 13 patients, five were frameshift mutations and three were nonsense mutations. 


\section{Identification of genetic features associated with poor pathological response and cancer recurrence}

With the determined mutation fingerprints of each patient, we next sought to find if these genetic features were associated with cancer recurrence and response to chemoradiotherapy. Logistic regression model was used to analyze the influence of single factor on pathological remission (less than $50 \%$ viable tumor cells, Table S2). Univariate analysis showed that Kelch-like ECH-associated protein 1 (KEAP1) mutation ( $15 \%$ vs. $0 \%, P=0.032)$, TMB-H ( $44 \%$ vs. $11 \%, P=0.007$ ) and TP53 polymorphism (rs $1042522 ; 37 \%$ vs. $14 \%, P=0.011$ ) were associated with poor pathological response (residual tumor cells $>50 \%$ ) in patients. The PIK3CA mutations had a trend to associate with poor pathological response (Fig. 2). Multivariate analysis revealed that TP53 polymorphism was the major factor associated with poor pathological response ( $P$-value 0.014$)$ and the TMB-H association with poor pathological response was partially independent from TP53 polymorphism ( $P$-value 0.14$)$. Due to co-occurrence with TP53 polymorphism, the association of KEAP1 mutations with pathological response was no longer significant with multivariate analysis ( $P$-value 0.78 ). Disease-free survival (DFS) analysis revealed that four genetic alterations were associated with poor DFS including KEAP1 mutation, SOX2-PIK3CA co-amplification, thymidylate synthase (TYMS) triple repeats (3R/3R) polymorphism and TERC amplification (Fig. 3 and S3). Analysis

of PIK3CA single/multiple mutations showed that they had no correlation with particular outcome of DFS, suggesting that compared to SOX2-PIK3CA co-amplification, activation of PI3K pathway by mutations was insufficient to predict clinical outcome of cervical cancer patients (Figure S4). Interestingly, KEAP1 mutation was found in both poor DFS group and poor chemoradiotherapy response group, further confirming the role of KEAP1-related signaling pathway in mediating poor disease outcome.

\section{Discussion}

In this study, we characterized genetic alteration of 62 cervical cancer cases in China and compared their molecular profile with that of Caucasian cervical cancer patients in TCGA database. PIK3CA was the most frequently mutated gene in cervical cancer regardless of racial groups, suggesting a universal dependence of cervical cancer on PI3K/AKT signal pathway. Both datasets highlighted three mutation hotspots in PIK3CA gene, including E542, E545 and H1047 which accounted for half of mutation sites. Interestingly, a number of genes' mutation frequency differed significantly in Chinese and Caucasian cervical cancer. The three genes predominantly mutated in Caucasian population were RPTOR, EGFR and TP53, all associated with PI3K/AKT pathway. The genes mainly mutated in Chinese patients were TERT, POLD1, NOS2 and FGFR3. TERT and POLD1 were associated with telomere maintenance in cells. It has been reported that human papillomavirus (HPV) type 16 E6 could activate TERT gene transcription[17], suggesting a close relationship between HPV infection and TERT expression. The TERT promoter mutations were found in 20\% cervical cancer in Indian population[18, 19], implying its prevalence in Asians. Gene amplification, rearrangement and protein expression of TERT were associated with poor clinical outcome in thyroid cancer, glioma and neuroblastoma[20] [21, 22]. Further clinical investigation is needed to evaluate the effect of TERT promoter mutation on survival of cervical cancer patients. Our finding of germline mutations enrichment in DNA repair pathway is in agreement with the germline mutation summary made by Bertelsen et. al.[23]. However, the mutation patterns of cervical cancer were 
different between two studies, likely due to larger patient cohort in our study and different racial/genetic background. Over $60 \%$ of germline mutations were truncation, frameshift or splicing variants deleterious to protein function, suggesting tumor suppressor role of these genes and importance of inactivation of DNA repair pathway in tumorigenesis. Several Poly (ADP-ribose) polymerase inhibitors (PARPi) have been approved in BRCA1/2-mutant ovarian and breast cancer[24]. Given the high mutation frequency of BRCA1/2 in cervical cancer, the clinical utility of PARPi in cervical cancer is worth exploration, especially in combination with chemotherapy or targeted therapy[24, 25]. The early onset of cancer was found in germline-mutant patients in this study, supporting the critical role of DNA repair gene mutations in carcinogenesis[26-28].

Although the neoadjuvant therapy to surgery is not a standard therapy for FIGO IB-IIA patients, neoadjuvant chemotherapy to surgery has been evaluated by two randomized phase III clinical trial (NCT00193739 and EORTC Protocol 55994, no results available yet)[6] and it was permitted in National Comprehensive Cancer Network (NCCN) 2020 guideline. The neoadjuvant brachytherapy and chemotherapy to radical hysterectomy was included in clinical practice guideline in China and has shown promising efficacy. It reduced the size of stage IB2-IIA cervical cancer and enabled radical surgery, achieving an overall survival comparable to standard chemo-radiation as well as a more favorable sideeffect profile. However, there were still $10 \%$ of patients whose tumor progressed after the treatment. One of the factors promoting tumor progression was poor response to neoadjuvant chemoradiotherapy. Identification of biomarkers predicting response to neoadjuvant therapy as well as tumor recurrence would enable early detection of recurrence and maximize therapeutic window for patients. Through univariate analysis, we found that TP53 polymorphism (rs1042522), KEAP1 mutation and TMB-H were associated with poor pathological response of tumor cells to neoadjuvant therapy. TP53 codon 72 arginine (polymorphism, rs1042522) has been shown to be more susceptible to HPV-related carcinogenesis[29] and more resistant to chemoradiotherapy in head and neck cancer [30]. Our data demonstrated that the association between TP53 polymorphism (P72R) and resistance of chemoradiotherapy also existed in cervical cancer, suggesting this correlation can potentially be generalized to other cancer types. In June 2020, Food and Drug Administration (FDA) expanded the approval of pembrolizumab (anti PD-1) to include any cancer with TMB-H. Our previous study has shown that the 10 mutations/Mb in the targeted panel possessed predictive value in patients response to immunotherapy[7]. The poor pathological response of TMB-H cervical cancer to chemoradiotherapy provided another rationale to apply immunotherapy to cervical cancer with TMB-H.

In our study, KEAP1 mutation, co-amplification of PIK3CA and SOX2, TERC amplification, and TYMS polymorphism were found associated with early onset of cancer recurrence. The KEAP1 mutation has been shown to increase radioresistance and local recurrence in lung squamous cell carcinoma[31]. Consistently, our pathological response and disease-free survival analysis revealed that KEAP1 mutation was also associated with chemo-radioresistance and local recurrence of cervical cancer in Chinese population. The human protein atlas showed that high KEAP1 expression was associated with prolonged survival of cervical cancer patients (291 cases) and was a favorable prognostic biomarker[32]. KEAP1 is an E3 ligase adaptor responsible for NRF2 ubiquitination and degradation. As NRF2 confers a survival 
advantage to tumors[33], KEAP1 mutations in cervical cancer likely reduced KEAP1 expression and function. Indeed, one of the two PD patients harboring KEAP1 mutations in our patient cohort had nonsense mutation at R260 in KEAP1 gene, abolishing its normal function and expression in tumor cells. Taken together, both our pathological response and DFS dataset, and the dataset from protein atlas indicated an importance of KEAP1 alteration in predicting survival of cervical cancer patients. The PIK3CA and SOX2 genes both are located on chromosome 3q26-28. Their co-amplification was critical for pathogenesis of squamous cell carcinoma[34] and was observed in $94 \%$ lung squamous cell carcinoma[35] and $100 \%$ esophageal squamous cell carcinoma[36]. Here we showed that coamplification of PIK3CA and SOX2 was associated with higher recurrence risk of cervical cancer.

In conclusion, we identified genetic alterations highly associated with Chinese cervical cancer patients and reported correlations of specific genetic features with poor chemo-radiotherapy response or early recurrence of cervical cancer in Chinese population. Future efforts should focus on validating these correlations in larger patient cohort and testing these potential biomarkers utility in clinical settings.

\section{Declarations}

\section{Ethics Approval and Consent to Participate}

This study was approved by the Institutional Review Board/Ethics Committee of Shandong Cancer Hospital and Institute. Written informed consent was obtained from each patient upon sample collection and for publication of the study.

\section{Funding}

This study was partially funded by Natural Science Foundation of China (NSFC81872475, NSFC81372413, NSFC81972683), National key Research and Development Program (2018YFC1313200), Shandong Natural Science Foundation Joint Fund (ZR2019LZL018), Shandong Key Research and Development Plan (2017CXGC1209 and 2017GSF18164) and the Outstanding Youth Natural Science Foundation of Shandong Province (JQ201423), Jinan Clinical Medicine Science and Technology Innovation Plan (201704095), National Key Research and Development Program of China (2016YFC0904700).

\section{Conflict of Interest:}

Zhenhao Fang, Qiuxiang Ou, Xue Wu, Jiani C. Yin, Jiaohui Pang, Xiaonan Wang, Yang Shao are employees of Nanjing Geneseeq Technology Inc. The remaining authors have nothing to declare.

\section{Data availability statement}

This study was based on the Chinese national cancer registry data. The authors do not own these data and hence are not permitted to share them in the original form (only in aggregate form, eg, publications). 
At the time of request data were provided by the Office for National Statistics but now all cancer registrations are owned and maintained by Public Health China.

\section{Consent for publication}

Not applicable.

\section{Contributions}

Yuchun Wei and Shuanghu Yuan designed the study. Yang Shao, Jinming Yu and Shuanghu Yuan supervised the study. Yuchun Wei, Chuqing Wei, Chen Liang and Ning Liu collected data. Dianbin Mu analyzed the pathological response. Yuchun Wei, Zhenhao Fang, Jiani C. Yin, Jiaohui Pang, Qiuxiang Ou, Xue Wu, Xiaonan Wang and Dianbin Mu analyzed data; Yuchun Wei, Zhenhao Fang, Jiani C. Yin, Jiaohui Pang and Qiuxiang Ou wrote the manuscript. All authors participated in data discussion and interpretation, and approved the final manuscript.

\section{References}

[1] Bray F, Ferlay J, Soerjomataram I, Siegel RL, Torre LA, Jemal A. Global cancer statistics 2018:

GLOBOCAN estimates of incidence and mortality worldwide for 36 cancers in 185 countries. CA: a cancer journal for clinicians. 2018;68:394-424.

[2] Small W, Jr., Bacon MA, Bajaj A, Chuang LT, Fisher BJ, Harkenrider MM, et al. Cervical cancer: A global health crisis. Cancer. 2017;123:2404-12.

[3] Chen W, Zheng R, Baade PD, Zhang S, Zeng H, Bray F, et al. Cancer statistics in China, 2015. CA: a cancer journal for clinicians. 2016;66:115-32.

[4] Marth C, Landoni F, Mahner S, McCormack M, Gonzalez-Martin A, Colombo N, et al. Cervical cancer: ESMO Clinical Practice Guidelines for diagnosis, treatment and follow-up. Annals of oncology : official journal of the European Society for Medical Oncology. 2017;28:iv72-iv83.

[5] Peters WA, 3rd, Liu PY, Barrett RJ, 2nd, Stock RJ, Monk BJ, Berek JS, et al. Concurrent chemotherapy and pelvic radiation therapy compared with pelvic radiation therapy alone as adjuvant therapy after radical surgery in high-risk early-stage cancer of the cervix. Journal of clinical oncology : official journal of the American Society of Clinical Oncology. 2000;18:1606-13.

[6] Ma Y, Zhao G, Qi J, Sun P, Liu C, Qu P, et al. Neoadjuvant brachytherapy and chemotherapy followed by radical surgery for stage IB2 and IIA cervical cancer: A retrospective comparison with chemoirradiation. Molecular and clinical oncology. 2018;8:617-22.

[7] Fang W, Ma Y, Yin JC, Hong S, Zhou H, Wang A, et al. Comprehensive Genomic Profiling Identifies Novel Genetic Predictors of Response to Anti-PD-(L)1 Therapies in Non-Small Cell Lung Cancer. Clinical cancer research : an official journal of the American Association for Cancer Research. 2019;25:5015-26. 
[8] Yang Z, Yang N, Ou Q, Xiang Y, Jiang T, Wu X, et al. Investigating Novel Resistance Mechanisms to Third-Generation EGFR Tyrosine Kinase Inhibitor Osimertinib in Non-Small Cell Lung Cancer Patients. Clinical cancer research : an official journal of the American Association for Cancer Research. 2018;24:3097-107.

[9] Shu Y, Wu X, Tong X, Wang X, Chang Z, Mao Y, et al. Circulating Tumor DNA Mutation Profiling by Targeted Next Generation Sequencing Provides Guidance for Personalized Treatments in Multiple Cancer Types. Scientific reports. 2017;7:583.

[10] Bolger AM, Lohse M, Usadel B. Trimmomatic: a flexible trimmer for Illumina sequence data. Bioinformatics. 2014;30:2114-20.

[11] Koboldt DC, Zhang Q, Larson DE, Shen D, McLellan MD, Lin L, et al. VarScan 2: somatic mutation and copy number alteration discovery in cancer by exome sequencing. Genome research. 2012;22:568-76.

[12] Newman AM, Bratman SV, Stehr H, Lee LJ, Liu CL, Diehn M, et al. FACTERA: a practical method for the discovery of genomic rearrangements at breakpoint resolution. Bioinformatics. 2014;30:3390-3.

[13] Yu G, He QY. ReactomePA: an R/Bioconductor package for reactome pathway analysis and visualization. Molecular bioSystems. 2016;12:477-9.

[14] Hellmann MD, Chaft JE, William WN, Jr., Rusch V, Pisters KM, Kalhor N, et al. Pathological response after neoadjuvant chemotherapy in resectable non-small-cell lung cancers: proposal for the use of major pathological response as a surrogate endpoint. The Lancet Oncology. 2014;15:e42-50.

[15] Tomczak K, Czerwinska P, Wiznerowicz M. The Cancer Genome Atlas (TCGA): an immeasurable source of knowledge. Contemporary oncology. 2015;19:A68-77.

[16] Fruman DA, Chiu H, Hopkins BD, Bagrodia S, Cantley LC, Abraham RT. The PI3K Pathway in Human Disease. Cell. 2017;170:605-35.

[17] McMurray HR, McCance DJ. Human papillomavirus type 16 E6 activates TERT gene transcription through induction of c-Myc and release of USF-mediated repression. Journal of virology. 2003;77:985261.

[18] Vinothkumar V, Arunkumar G, Revathidevi S, Arun K, Manikandan M, Rao AK, et al. TERT promoter hot spot mutations are frequent in Indian cervical and oral squamous cell carcinomas. Tumour biology : the journal of the International Society for Oncodevelopmental Biology and Medicine. 2016;37:7907-13.

[19] Annunziata C, Pezzuto F, Greggi S, Ionna F, Losito S, Botti G, et al. Distinct profiles of TERT promoter mutations and telomerase expression in head and neck cancer and cervical carcinoma. International journal of cancer. 2018;143:1153-61. 
[20] Yuan X, Larsson C, Xu D. Mechanisms underlying the activation of TERT transcription and telomerase activity in human cancer: old actors and new players. Oncogene. 2019;38:6172-83.

[21] Liu T, Yuan X, Xu D. Cancer-Specific Telomerase Reverse Transcriptase (TERT) Promoter Mutations: Biological and Clinical Implications. Genes. 2016;7.

[22] Valentijn LJ, Koster J, Zwijnenburg DA, Hasselt NE, van Sluis P, Volckmann R, et al. TERT rearrangements are frequent in neuroblastoma and identify aggressive tumors. Nature genetics. 2015;47:1411-4.

[23] Bertelsen B, Tuxen IV, Yde CW, Gabrielaite M, Torp MH, Kinalis S, et al. High frequency of pathogenic germline variants within homologous recombination repair in patients with advanced cancer. NPJ genomic medicine. 2019;4:13.

[24] Yi M, Dong B, Qin S, Chu Q, Wu K, Luo S. Advances and perspectives of PARP inhibitors. Experimental hematology \& oncology. 2019;8:29.

[25] Yap TA, Kristeleit R, Michalarea V, Pettitt SJ, Lim JSJ, Carreira S, et al. Phase I trial of the poly(ADPribose) polymerase (PARP) inhibitor olaparib and AKT inhibitor capivasertib in patients with BRCA1/2 and non-BRCA1/2 mutant cancers. Cancer discovery. 2020.

[26] Wagner JE, Tolar J, Levran O, Scholl T, Deffenbaugh A, Satagopan J, et al. Germline mutations in BRCA2: shared genetic susceptibility to breast cancer, early onset leukemia, and Fanconi anemia. Blood. 2004;103:3226-9.

[27] Dite GS, Jenkins MA, Southey MC, Hocking JS, Giles GG, McCredie MR, et al. Familial risks, earlyonset breast cancer, and BRCA1 and BRCA2 germline mutations. Journal of the National Cancer Institute. 2003;95:448-57.

[28] Yang X, Leslie G, Doroszuk A, Schneider S, Allen J, Decker B, et al. Cancer Risks Associated With Germline PALB2 Pathogenic Variants: An International Study of 524 Families. Journal of clinical oncology : official journal of the American Society of Clinical Oncology. 2020;38:674-85.

[29] Storey A, Thomas M, Kalita A, Harwood C, Gardiol D, Mantovani F, et al. Role of a p53 polymorphism in the development of human papillomavirus-associated cancer. Nature. 1998;393:229-34.

[30] Bergamaschi D, Gasco M, Hiller L, Sullivan A, Syed N, Trigiante G, et al. p53 polymorphism influences response in cancer chemotherapy via modulation of p73-dependent apoptosis. Cancer cell. 2003;3:387402 .

[31] Jeong Y, Hoang NT, Lovejoy A, Stehr H, Newman AM, Gentles AJ, et al. Role of KEAP1/NRF2 and TP53 Mutations in Lung Squamous Cell Carcinoma Development and Radiation Resistance. Cancer discovery. 2017;7:86-101. 
[32] Uhlen M, Zhang C, Lee S, Sjostedt E, Fagerberg L, Bidkhori G, et al. A pathology atlas of the human cancer transcriptome. Science. 2017;357.

[33] Robledinos-Anton N, Fernandez-Gines R, Manda G, Cuadrado A. Activators and Inhibitors of NRF2: A Review of Their Potential for Clinical Development. Oxidative medicine and cellular longevity.

2019;2019:9372182.

[34] Kim BR, Van de Laar E, Cabanero M, Tarumi S, Hasenoeder S, Wang D, et al. SOX2 and PI3K Cooperate to Induce and Stabilize a Squamous-Committed Stem Cell Injury State during Lung Squamous Cell Carcinoma Pathogenesis. PLoS biology. 2016;14:e1002581.

[35] Cancer Genome Atlas Research N. Comprehensive genomic characterization of squamous cell lung cancers. Nature. 2012;489:519-25.

[36] Dai H, Shao YW, Tong X, Wu X, Pang J, Feng A, et al. YAP1 amplification as a prognostic factor of definitive chemoradiotherapy in nonsurgical esophageal squamous cell carcinoma. Cancer medicine. 2020;9:1628-37.

\section{Figures}

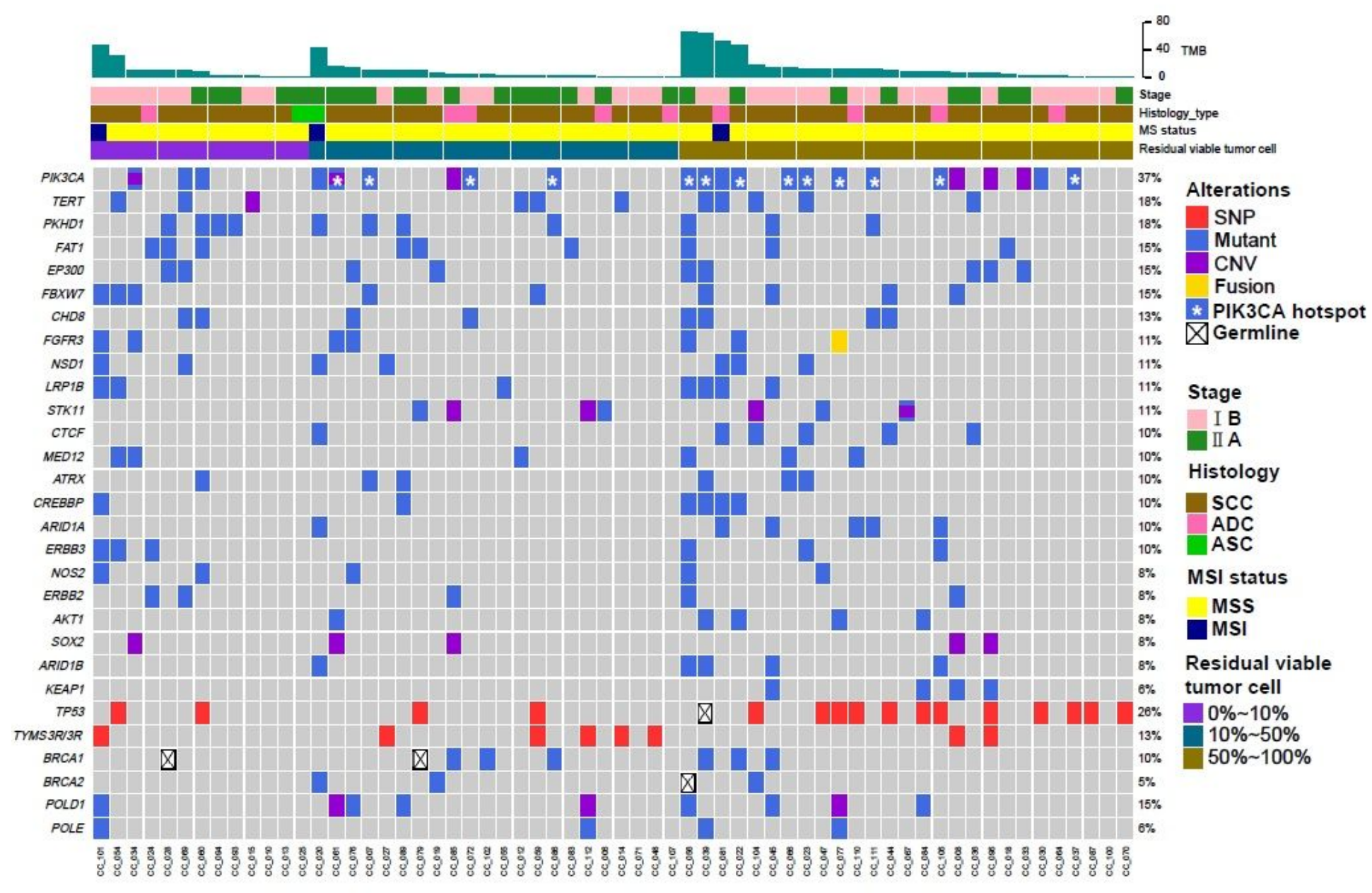


Figure 1

Distribution of gene alterations correlated with pathological response. Gene alterations and patient clinical characteristics were shown at the top and bottom, respectively. Patients were separated into three groups, of which H\&E stains exhibited $<10 \%, 10-50 \%$ and $50 \%-100 \%$ viable tumor cells. The BRCA $1 / 2$, POLD1 and POLE were genes related to targeted therapy or immunotherapy. PIK3CA hotspot mutations on $\mathrm{E} 542$, E545 and $\mathrm{H} 1047$ were marked by white asterisks. The $0-10 \%, 10-50 \%$ and $50-100 \%$ viable tumor cells represent major, partial and poor pathological response, respectively. SCC, squamous cell carcinoma. ADC, adenocarcinoma. ASC, adenosquamous carcinoma. MSI, microsatellite instability. MSS, microsatellite stability.

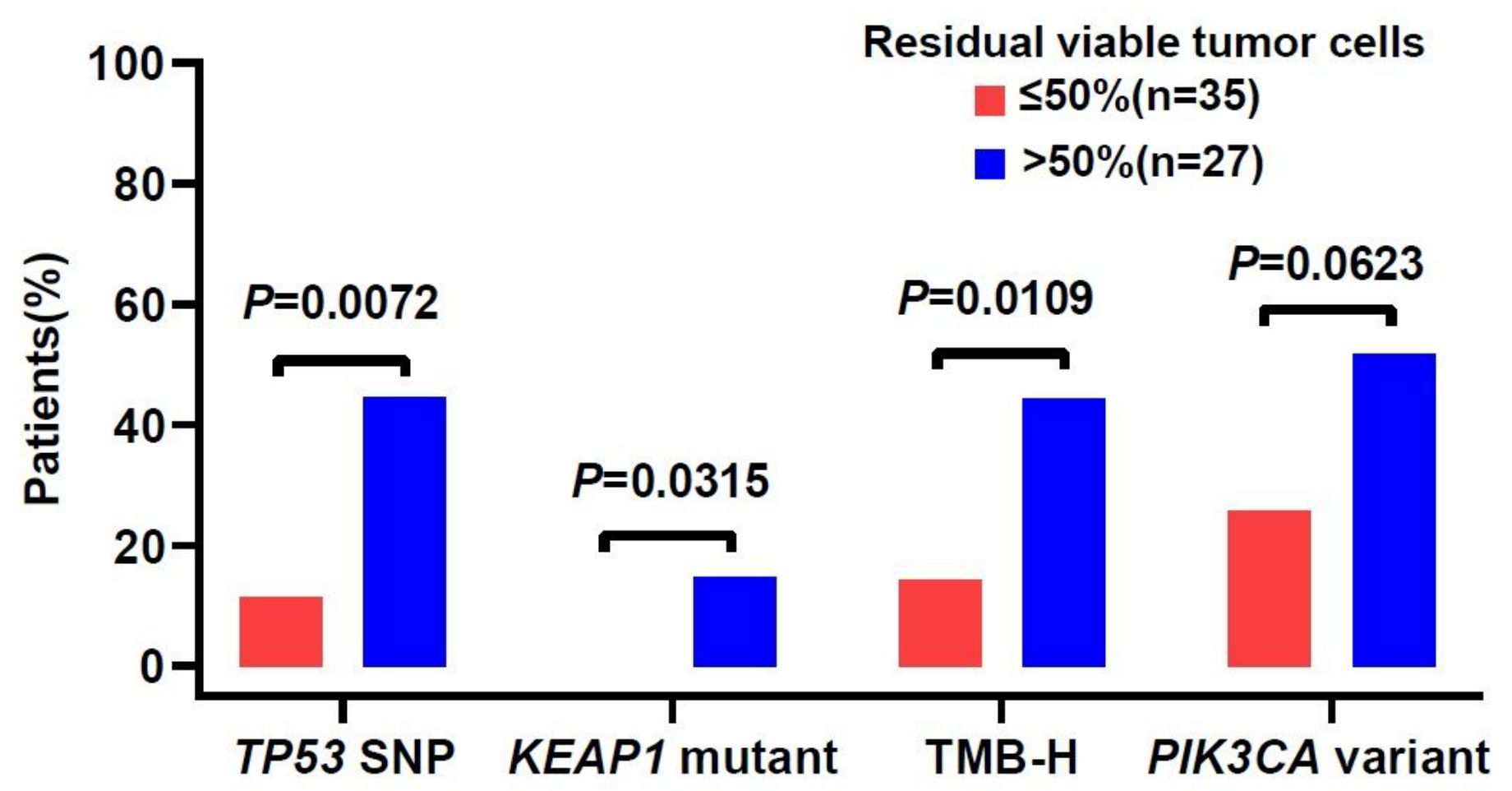

Figure 2

Association of TP53 polymorphism, KEAP1, PIK3CA mutations and high TMB with poor pathological response to neoadjuvant chemoradiotherapy. The TP53 single nucleotide polymorphism (SNP), KEAP1, PIK3CA mutation and high tumor mutation burden (TMB-H) were enriched in poor pathological response group with more than $50 \%$ viable tumor cells (in blue). 

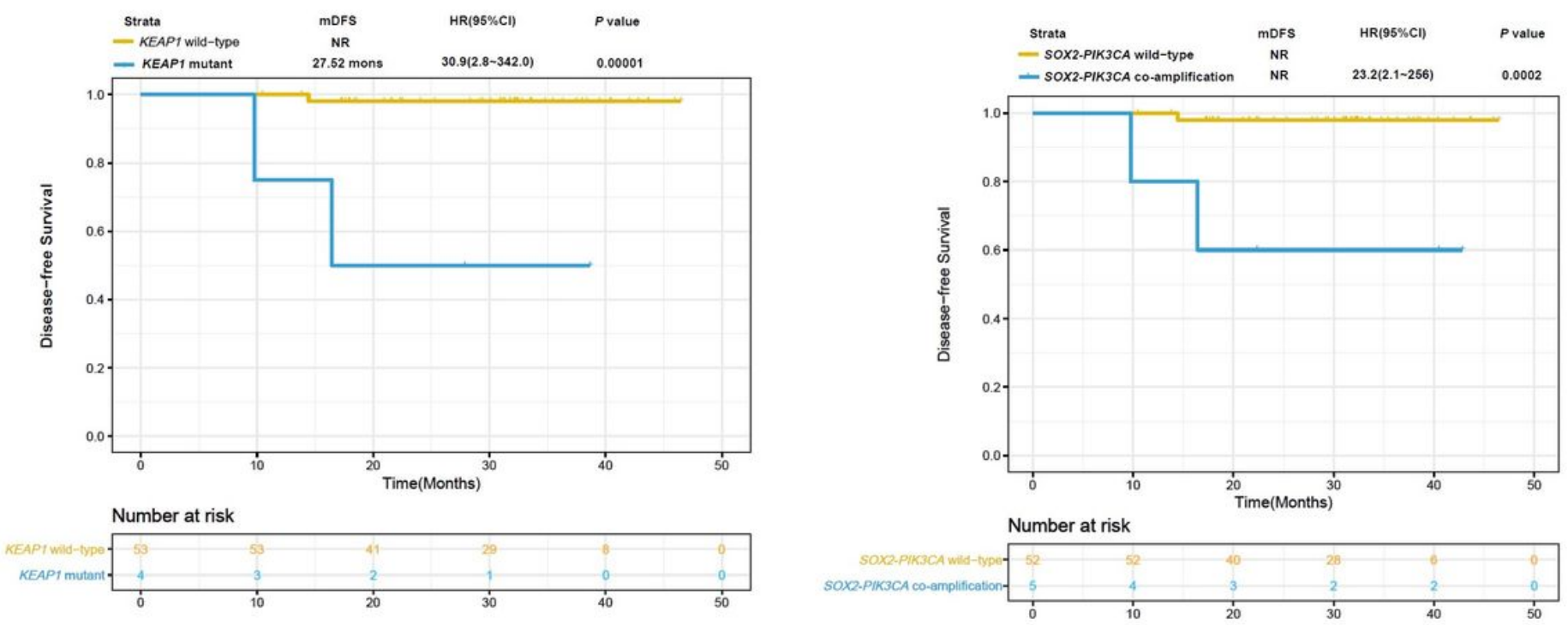

Figure 3

Association of KEAP1 mutation and SOX2-PIK3CA co-amplification with high cervical cancer recurrence risk. Poor DFS was observed in patients harbouring KEAP1 mutation (A) or SOX2-PIK3CA co-amplification (B).

\section{Supplementary Files}

This is a list of supplementary files associated with this preprint. Click to download.

- Supplementals20200903v2.pptx 